\title{
ON STABILITY OF A CATENOIDAL LIQUID BRIDGE
}

\author{
LIANMIN ZHOU
}

\begin{abstract}
We derive a stability criterion for a catenoidal liquid bridge making contact angles $\gamma_{1}$ and $\gamma_{2}$ with two parallel plates. We show that for the case of equal contact angles $\gamma_{1}=\gamma_{2}=\gamma$ the stability and instability sets are connected on the interval of admissible $\gamma$. We also give an example showing that for unequal contact angles, the family of stable catenoidal drops with one contact angle fixed can be disconnected with respect to the other angle. At the end of the paper we give a complete description of the stability and instability sets for various contact angles.
\end{abstract}

\section{Introduction.}

A liquid bridge joining two parallel homogeneous plates in the absence of gravity will always take the form of a catenoid, nodoid or unduloid. We restrict attention here to catenoidal bridges, and ask under what condition the bridge will be stable in the sense of providing a local energy minimum.

Given the contact angles $\gamma_{1}, \gamma_{2}$ of the plates and the separation distance $h$ between them, it turns out that the volume of the liquid drop plays a crucial role in the stability of the bridge. Since we are only concerned with catenoidal liquid bridges in this paper, the volume is entirely determined by $\gamma_{1}, \gamma_{2}$ and $h$. We assume that $h=1$ by scaling properly. Therefore it entirely depends upon the contact angles of the plates whether or not a stable catenoidal liquid bridge will be formed.

In the first part of this paper, we will derive a stability criterion for a catenoidal liquid drop making contact angels $\gamma_{1}, \gamma_{2}$. We show that such a bridge is stable if $F\left(\gamma_{1}, \gamma_{2}\right)<0$ and unstable if $F\left(\gamma_{1}, \gamma_{2}\right)>0$, where

$$
F\left(\gamma_{1}, \gamma_{2}\right) \stackrel{\text { def }}{=} 5 \int_{\gamma_{1}}^{\pi-\gamma_{2}} \frac{1}{\sin ^{5} \theta} d \theta \int_{\gamma_{1}}^{\pi-\gamma_{2}} \frac{1}{\sin \theta} d \theta-9\left(\int_{\gamma_{1}}^{\pi-\gamma_{2}} \frac{1}{\sin ^{3} \theta} d \theta\right)^{2}
$$

In the second part, we would like to understand what the above stability criterion really says about the contact angles, at least in the special case of equal contact angles. We show that there exists $\gamma_{0}$ such that a stable catenoidal liquid bridge making equal contact angles $\gamma$ with the plates will 
be formed if $\gamma>\gamma_{0}$ and such a bridge will not be formed if $\gamma<\gamma_{0}$. In other words, the $\gamma$-sets of stability and instability are connected. This result is obtained by showing that the following transcedental equation has one and only one solution:

$$
F(\gamma, \gamma) \stackrel{\text { def }}{=} 4\left[5 \int_{\gamma}^{\frac{\pi}{2}} \frac{1}{\sin ^{5} \theta} d \theta \int_{\gamma}^{\frac{\pi}{2}} \frac{1}{\sin \theta} d \theta-9\left(\int_{\gamma}^{\frac{\pi}{2}} \frac{1}{\sin ^{3} \theta} d \theta\right)^{2}\right]
$$

Finally we would like to mention an interesting question raised by Finn and Vogel [7, pages 17-18]: if a bridge is unstable, then extending that Delaunay surface will only result in unstable bridges? In other words, if one liquid bridge is a part of another stable liquid bridge, must it also be stable? They have pointed out that the requirement that the perturbation be volume preserving makes the problem nontrivial. They also point out that if this statement failed, it must be due to the second cause of instability in Vogel's stability criterion.

An affirmative answer to this question looks more natural. An affirmative answer would imply that a catenoidal liquid bridge (making equal contact angles) is stable if $\gamma>\gamma_{0}$ and is unstable if $\gamma<\gamma_{0}$ and therefore there is no need of showing that (2) has only one zero. Recently we have found an example that the answer to the above question is not always affirmative. On the one hand, this shows that for unequal contact angles, the stability set can be disconnected, and on the other hand, this result sets into relief the need for an analytic proof that (2) has only one zero in the equal angle case.

The stability problem of a catenoidal liquid bridge making equal contact angles with the plates has previously been studied by several other authors. In [8], Langbein derived a stability criterion equivalent to (2) above using Vogel's minimum volume criterion and it is he who first computed the numerical value of $\gamma_{0}$ as $14.97^{\circ}$. Strube $[\mathbf{1 0}, \mathbf{1 1}]$ derived his stability criterion for a catenoidal liquid bridge directly from first principles. Since we were not aware of Strube's previous work, we took the more complicated approach similar to Strube's.

Acknowledgment. I would like to take this opportunity to thank the referee for bringing to my attention the previous work of Strube and for several valuable suggestions which have led to much improvement of this paper. I would also like to thank Robert Finn for many helpful discussions and constant encouragement. 


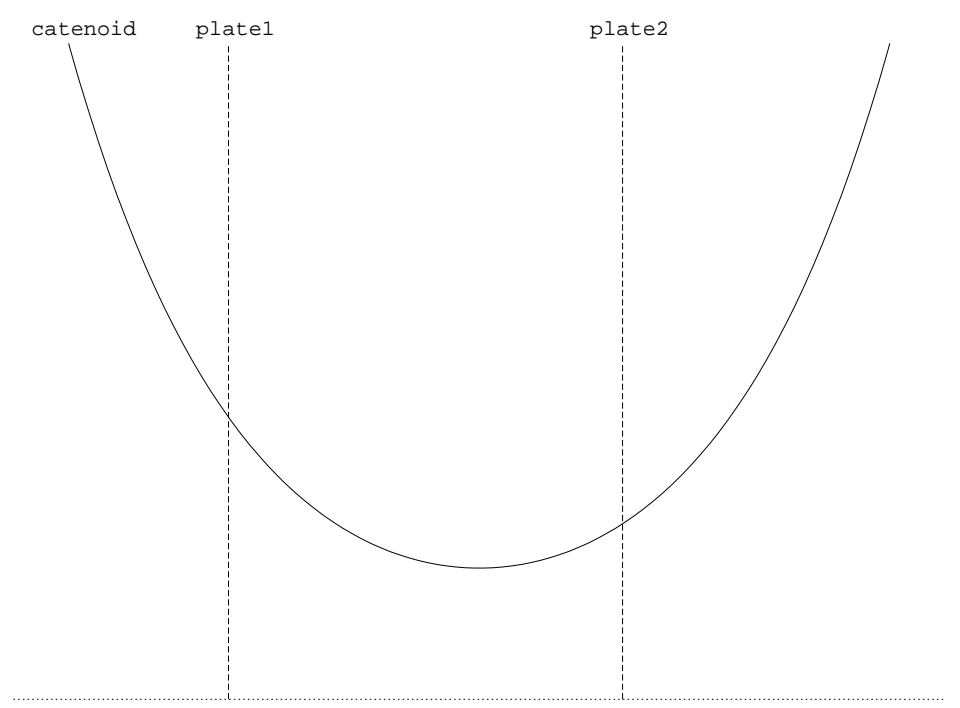

Figure 1. A catenoid.

\section{Vogel's Stability Criterion.}

Let $f(u), 0 \leq u \leq h$ denote the profile curve of a liquid drop trapped between two parallel (vertical) plates located at $x=0$ and $x=h$. Then $f$ is a solution of the following Euler-Lagrange equation:

$$
\begin{gathered}
-\frac{1}{2}\left(\frac{f^{\prime \prime}}{\left(1+\left(f^{\prime}\right)^{2}\right)^{3 / 2}}-\frac{1}{f\left(1+\left(f^{\prime}\right)^{2}\right)^{1 / 2}}\right)=H \\
f^{\prime}(0)=-\cot \gamma_{1} \quad f^{\prime}(h)=\cot \gamma_{2}
\end{gathered}
$$

where $\mathrm{H}$ is the mean curvature of the surface and $\gamma_{1}, \gamma_{2}$ are the contact angles that the surface makes with the two plates.

Suppose that the following Sturm-Liouville problem associated with (3)

$$
\begin{gathered}
L(\psi) \equiv-\left(\frac{f \psi^{\prime}}{\left(1+\left(f^{\prime}\right)^{2}\right)^{3 / 2}}\right)^{\prime}-\frac{\psi}{f\left(1+\left(f^{\prime}\right)^{2}\right)^{1 / 2}}=\lambda \psi \\
\psi^{\prime}(0)=\psi^{\prime}(h)=0
\end{gathered}
$$

has only one non-positive eigenvalue. Then

1. the drop is stable if $\int_{0}^{h} \phi(u) f(u) d u<0$ where $\phi(u) \in C^{2}([0, h])$ satisfies $L(\phi)=f$ with $\phi^{\prime}(0)=\phi^{\prime}(h)=0$ and

2. the drop is unstable if $\int_{0}^{h} \phi(u) f(u) d u>0$ where $\phi$ is the same as above. 


\section{Stability and Instability of Catenoidal Surfaces.}

When the mean curvature $H=0$, the solution of (3) is a catenoid

$$
f(u)=a \cosh \left(\frac{u-u_{0}}{a}\right), 0 \leq u \leq h
$$

where the constant $a$ and the center $u_{0}$ are uniquely determined by the separation distance $h$ of the plates and the contact angles $\gamma_{1}$ and $\gamma_{2}$. Theorem 1 is a special case of Lemma 3.4 in Vogel [13] and the proof follows that of Theorem 5.7 in Finn and Vogel [7].

Theorem 1. Let $f(u)=a \cosh \left[\left(u-u_{0}\right) / a\right], 0 \leq u \leq h$ be the profile curve of the catenoidal drop making contact angles $\gamma_{1}$ and $\gamma_{2}$ with the plates. Then for any $a>0$ and any $u_{0}$ (and therefore for any separation distance $h$ and any contact angles $\left.\gamma_{1}, \gamma_{2}\right)$, the associated Sturm-Liouville problem (4) has one and only one non-positive eigenvalue.

Theorem 2. Let $f(u)=a \cosh \left[\left(u-u_{0}\right) / a\right], 0 \leq u \leq h$ be the profile curve of the catenoidal drop making contact angles $\gamma_{1}$ and $\gamma_{2}$ with the plates, as in (5). Suppose that $\phi(u) \in C^{2}([0, h])$ solves $L(\phi)=f$ with $\phi^{\prime}(0)=\phi^{\prime}(h)=0$ and $F\left(\gamma_{1}, \gamma_{2}\right)$ as defined in (1). Then

$$
\int_{0}^{h} \phi(u) f(u) d u=a^{3} F\left(\gamma_{1}, \gamma_{2}\right)\left[\int_{\gamma_{1}}^{\pi-\gamma_{2}} \frac{1}{\sin \theta} d \theta\right]^{-1}
$$

Theorem 3. Suppose that $F\left(\gamma_{1}, \gamma_{2}\right)$ is as in Theorem 2 and $\gamma_{0} \approx 14.97^{\circ}$ is defined as in (2). Then $F(\gamma, \gamma)<0$ if $\gamma>\gamma_{0}$ and $F(\gamma, \gamma)>0$ if $\gamma<\gamma_{0}$.

Corollary 4. Suppose that a catenoidal drop makes contact angles $\gamma_{1}$ and $\gamma_{2}$ with the plates.

1. This drop is stable if $F\left(\gamma_{1}, \gamma_{2}\right)<0$ and is unstable if $F\left(\gamma_{1}, \gamma_{2}\right)>0$;

2. In the case that the two contact angles $\gamma_{1}=\gamma_{2}=\gamma$, the drop is stable if $\gamma>\gamma_{0}$ and unstable if $\gamma<\gamma_{0}$.

\section{Proof of Theorems.}

A) Proof of Theorem 1. Theorem 1 is a special case of Lemma 3.4 of [13] and the proof of this theorem uses the idea of the proof of Theorem 5.7 in [7]. We prove Theorem 1 in two steps: 
In Lemma 1, we show that, when the two plates are placed close enough to each other, the Sturm-Liouville problem associated with a catenoid has

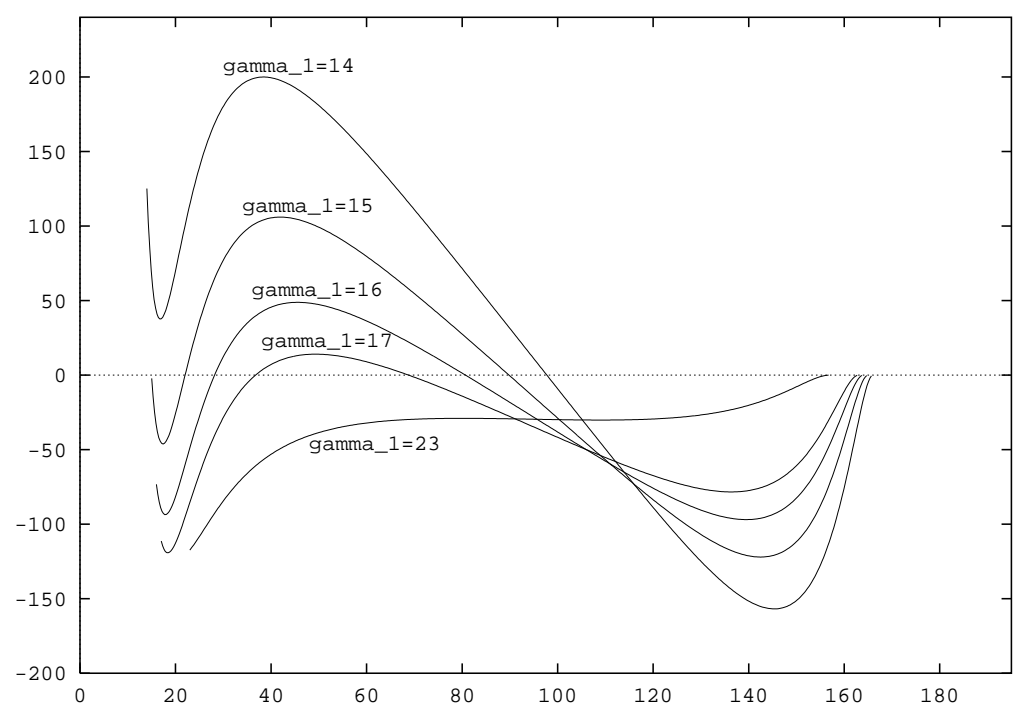

Figure 2. Some graphs of $F\left(\gamma_{1}, \gamma_{2}\right)$ w.r.t. $\gamma_{2}$.

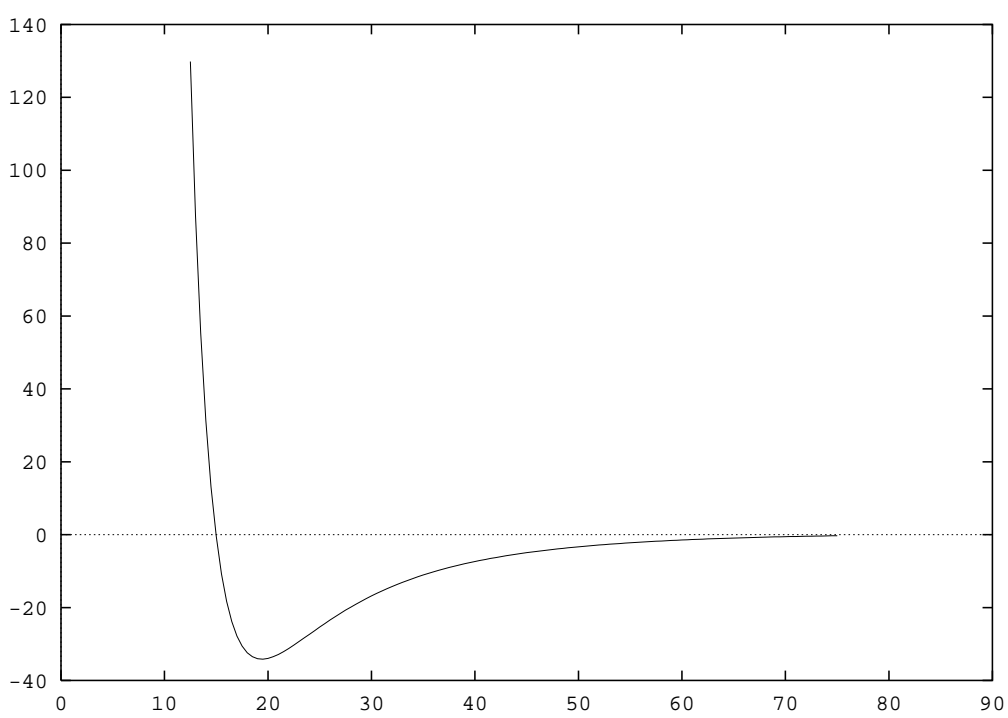

Figure 3. The graph of $F(\gamma, \gamma)$.

only one non-positive eigenvalue. In Lemma 2, we proceed to argue that it still has only one non-positive eigenvalue when the two plates are moved 
apart from each other by showing that it never has 0 as one of its eigenvalues wherever the plates are.

Lemma 1. Let $f(u)=a \cosh \left[\left(u-u_{0}\right) / a\right], 0 \leq u \leq h$ be defined as in (5). Suppose that $a$ is fixed and that the two plates are placed close enough, i.e. $h$ is small enough or equivalently, $\gamma_{1}$ is close enough to $\pi-\gamma_{2}$. Then the liquid drop is stable and the associated Sturm-Liouville problem (4) has only one non-positive eigenvalue.

Proof. We will show that $B(\psi) \equiv(L(\psi), \psi)>0$ for all $\psi \in f^{\perp}$, where

$$
f^{\perp} \equiv\left\{\psi \in C^{2}:\|\psi\|_{2}=1 \text { and } \int_{0}^{h^{\prime}} f \psi d u=0\right\} .
$$

Indeed, suppose that $f \geq A>0$ and $\left|f^{\prime}\right| \leq B$. Then

$$
\begin{aligned}
B(\psi) & =\int_{0}^{h}\left(\frac{f\left(\psi^{\prime}\right)^{2}}{\left(1+\left(f^{\prime}\right)^{2}\right)^{3 / 2}}-\frac{\psi^{2}}{f\left(1+\left(f^{\prime}\right)^{2}\right)^{1 / 2}}\right) d u>\int_{0}^{h}\left(\frac{A\left(\psi^{\prime}\right)^{2}}{\left(1+B^{2}\right)^{3 / 2}}-\frac{\psi^{2}}{A}\right) d u \\
& =C_{1} \int_{0}^{h}\left[\left(\psi^{\prime}\right)^{2}-C_{2} \psi^{2}\right] d u=C_{1}\left[\int_{0}^{h}\left(\psi^{\prime}\right)^{2} d u-C_{2}\right]
\end{aligned}
$$

where $C_{1}$ and $C_{2}$ are two postive constants.

Since $\psi \in f^{\perp}$ and $\|\psi\|_{2}$, we may assume that there exist $u_{1}$ and $u_{2}$ such that $\psi\left(u_{1}\right)<0$ and $\psi\left(u_{2}\right)>1$. Thus $\int_{0}^{h}\left|\psi^{\prime}(u)\right| d u>1$. Therefore

$$
\frac{1}{h} \int_{0}^{h}\left|\psi^{\prime}\right|^{2} d u \geq\left[\frac{1}{h} \int_{0}^{h}\left|\psi^{\prime}\right| d u\right]^{2}>\frac{1}{h^{2}} .
$$

If $h$ is small enough, then $\int_{0}^{h}\left(\psi^{\prime}\right)^{2} d u>1 / h>C_{2}$. This, together with (6), implies that $B(\psi)>0$ if $h$ is small enough. Therefore, by a result of Vogel, the drop is stable and the associated Sturm-Liouville problem has only one non-positive eigenvalue.

Lemma 2. The Sturm-Liouville problem (4) associated with a catenoid cannot have 0 as one of its eigenvalues, regardless where the two plates are placed.

Proof. We prove this lemma by contradiction. Suppose that zero were an eigenvalue of the Sturm-Liouville problem (4) and $\psi$ its eigenfunction which is not identically 0 . Then $\psi$ solves the following problem

$$
\begin{gathered}
-\left(\frac{f \psi^{\prime}}{\left(1+\left(f^{\prime}\right)^{2}\right)^{3 / 2}}\right)^{\prime}-\frac{\psi}{f\left(1+\left(f^{\prime}\right)^{2}\right)^{1 / 2}}=0 \\
\psi^{\prime}(0)=\psi^{\prime}(h)=0 .
\end{gathered}
$$


Now we place $f(u)=a \cosh \left[\left(u-u_{0}\right) / a\right]$ into (7) about and obtain

$-a^{2} \cosh \frac{u-u_{0}}{a} \psi^{\prime \prime}(u)+2 a \sinh \frac{u-u_{0}}{a} \psi^{\prime}(u)-\cosh \frac{u-u_{0}}{a} \psi(u)=0$

$$
\psi^{\prime}(0)=\psi^{\prime}(h)=0
$$

It is easy to check that the ODE (8) has the following general solution

$$
\psi=c_{1} \sinh \frac{u-u_{0}}{a}+c_{2}\left(u \sinh \frac{u-u_{0}}{a}-a \cosh \frac{u-u_{0}}{a}\right)
$$

where $c_{1}$ and $c_{2}$ are constants to be determined. Subject to the boundary condition (9), we must have $c_{1}=c_{2}=0$. Therefore $\psi \equiv 0$, a contradiction.

Since we know that the smallest eigenvalue of the Sturm-Liouville problem is always less than 0 , this completes the proof of Theorem 1.

B) Proof of Theorem 2 . Placing $f(u)=a \cosh \left[\left(u-u_{0}\right) / a\right]$ into the boundary problem $L(\phi)=f$ with $\phi^{\prime}(0)=\phi^{\prime}(h)=0$, we obtain

$$
a^{2} \phi^{\prime \prime}(u)-2 a \tanh \frac{u-u_{0}}{a} \phi^{\prime}(u)+\phi(u)=-a^{2} \cosh ^{3} \frac{u-u_{0}}{a}
$$

with $\phi^{\prime}(0)=\phi^{\prime}(h)=0$. Solving the above boundary problem yields

$$
\begin{array}{r}
\phi(u)=\sinh \frac{u-u_{0}}{a} \int_{0}^{u}\left(t \sinh \frac{t-u_{0}}{a}-a \cosh \frac{t-u_{0}}{a}\right) \cosh \frac{t-u_{0}}{a} d t- \\
\left(u \sinh \frac{u-u_{0}}{a}-a \cosh \frac{u-u_{0}}{a}\right)\left(\frac{a}{2} \cosh ^{2} \frac{u-u_{0}}{a}-c_{2}\right)
\end{array}
$$

where $c_{2}=a / 2 \cosh ^{2}\left(h-u_{0}\right) / a-(1 / h) \int_{0}^{h}\left(u \sinh \left(u-u_{0}\right) / a-a \cosh (u-\right.$ $\left.\left.u_{0}\right) / a\right) \cosh \left(u-u_{0}\right) / a d u$.

After some straightforward (but tedious) calculation and simplification, we obtain

$$
\int_{0}^{h} \phi(u) f(u) d u=\frac{5 a^{3}}{4} \int_{0}^{h} \cosh ^{4} \frac{u-u_{0}}{a} d u-\frac{9 a^{3}}{4 h}\left(\int_{0}^{h} \cosh ^{2} \frac{u-u_{0}}{a} d u\right)^{2} .
$$

We now make the transformation $\cot \theta=-\sinh \left[\left(u-u_{0}\right) / a\right]$ and note that $f^{\prime}(0)=-\cot \gamma_{1}$ and $f^{\prime}(h)=\cot \gamma_{2}$. It follows from (10) that $\int_{0}^{h} \phi(u) f(u) d u$ is equal to

$$
\frac{a^{3}}{\int_{\gamma_{1}}^{\pi-\gamma_{2}} \frac{1}{\sin \theta} d \theta}\left\{5 \int_{\gamma_{1}}^{\pi-\gamma_{2}} \frac{1}{\sin ^{5} \theta} d \theta \int_{\gamma_{1}}^{\pi-\gamma_{2}} \frac{1}{\sin \theta} d \theta-9\left(\int_{\gamma_{1}}^{\pi-\gamma_{2}} \frac{1}{\sin ^{3} \theta} d \theta\right)^{2}\right\}
$$


C) Proof of Theorem 3. For convenience, we denote

$$
g(\gamma)=5 \int_{\gamma}^{\frac{\pi}{2}} \frac{1}{\sin ^{5} \theta} d \theta \int_{\gamma}^{\frac{\pi}{2}} \frac{1}{\sin \theta} d \theta-9\left(\int_{\gamma}^{\frac{\pi}{2}} \frac{1}{\sin ^{3} \theta} d \theta\right)^{2} .
$$

Differentiating (11) with respect to $\gamma$, we obtain

$$
\begin{aligned}
g^{\prime}(\gamma)= & -\frac{5}{\sin ^{5} \gamma} \int_{\gamma}^{\frac{\pi}{2}} \frac{d \theta}{\sin \phi}-\frac{5}{\sin \gamma} \int_{\gamma}^{\frac{\pi}{2}} \frac{d \theta}{\sin ^{5} \phi}+\frac{18}{\sin ^{3} \gamma} \int_{\gamma}^{\frac{\pi}{2}} \frac{d \theta}{\sin ^{3} \phi} \\
= & \frac{1}{\sin ^{5} \gamma}\left[\left(-5-\frac{15}{8} \sin ^{4} \gamma+9 \sin ^{2} \gamma\right)\left(-\ln \tan \frac{\gamma}{2}\right)\right. \\
& \left.\quad+\left(\frac{31}{4}-\frac{15}{8} \sin ^{2} \gamma\right) \cos \gamma\right] \\
= & \frac{1}{\sin ^{5} \gamma}\left[h_{1}(\gamma)+h_{2}(\gamma)\right] .
\end{aligned}
$$

where $h_{1}(\gamma)=\left(-5-\frac{15}{8} \sin ^{4} \gamma+9 \sin ^{2} \gamma\right)\left(-\ln \tan \frac{\gamma}{2}\right)$ is an increasing function on $\left(0,50^{\circ}\right)$ and $h_{2}(\gamma)=\left(\frac{31}{4}-\frac{15}{8} \sin ^{2} \gamma\right) \cos \gamma$ is a decreasing function. We wish to show that $g(\gamma)$ has a unique zero at $\gamma_{0}$ and that $g(\gamma)>0$ on the interval $\left(0, \gamma_{0}\right)$ and $g(\gamma)<0$ on the interval $\left(\gamma_{0}, \pi / 2\right)$.

i) If $50^{\circ} \leq \gamma<90^{\circ}$,

$$
\begin{aligned}
g(\gamma) & \leq \frac{5}{\sin ^{2} \gamma} \int_{\gamma}^{\frac{\pi}{2}} \frac{1}{\sin ^{3} \theta} d \theta \int_{\gamma}^{\frac{\pi}{2}} \frac{1}{\sin \theta} d \theta-9 \int_{\gamma}^{\frac{\pi}{2}} \frac{1}{\sin ^{3} \theta} d \theta \int_{\gamma}^{\frac{\pi}{2}} \frac{1}{\sin \theta} d \theta \\
& =\left(\frac{5}{\sin ^{2} \gamma}-9\right) \int_{\gamma}^{\frac{\pi}{2}} \frac{d \theta}{\sin ^{3} \phi} \int_{\gamma}^{\frac{\pi}{2}} \frac{d \theta}{\sin \phi} \\
& \leq\left(\frac{5}{\sin ^{2} 50^{\circ}}-9\right) \int_{\gamma}^{\frac{\pi}{2}} \frac{d \theta}{\sin ^{3} \phi} \int_{\gamma}^{\frac{\pi}{2}} \frac{d \theta}{\sin \phi} \\
& \approx-0.4796 \int_{\gamma}^{\frac{\pi}{2}} \frac{d \theta}{\sin ^{3} \phi} \int_{\gamma}^{\frac{\pi}{2}} \frac{d \theta}{\sin \phi}<0 .
\end{aligned}
$$

ii) If $29^{\circ} \leq \gamma \leq 50^{\circ}$,

$$
g^{\prime}(\gamma) \geq \frac{1}{\sin ^{5} \gamma}\left(h_{1}\left(29^{\circ}\right)+h_{2}\left(50^{\circ}\right)\right)>\frac{1}{\sin ^{5} \gamma}(-4.042+4.274)>0 .
$$

iii) If $22^{\circ} \leq \gamma \leq 29^{\circ}$, then

$$
g^{\prime}(\gamma) \geq \frac{1}{\sin ^{5} \gamma}\left(h_{1}\left(22^{\circ}\right)+h_{2}\left(29^{\circ}\right)\right)>\frac{1}{\sin ^{5} \gamma}(-6.182+6.392)>0 .
$$

iv) If $20^{\circ} \leq \gamma \leq 22^{\circ}$, then

$$
g^{\prime}(\gamma) \geq \frac{1}{\sin ^{5} \gamma}\left(h_{1}\left(20^{\circ}\right)+h_{2}\left(22^{\circ}\right)\right)>\frac{1}{\sin ^{5} \gamma}(-6.895+6.941)>0 .
$$


v) If $19.5^{\circ} \leq \gamma \leq 20^{\circ}$, then

$$
\begin{aligned}
g(\gamma) & \leq 5 \int_{19.5^{\circ}}^{90^{\circ}} \frac{d \theta}{\sin ^{5} \phi} \int_{19.5^{\circ}}^{90^{\circ}} \frac{d \theta}{\sin \phi}-9\left(\int_{20^{\circ}}^{90^{\circ}} \frac{d \theta}{\sin ^{5} \phi}\right)^{2} \\
& <200.899-214.703<0 .
\end{aligned}
$$

vi) If $19^{\circ} \leq \gamma \leq 19.5^{\circ}$, then

$$
\begin{aligned}
g(\gamma) & \leq 5 \int_{19^{\circ}}^{90^{\circ}} \frac{d \theta}{\sin ^{5} \phi} \int_{19^{\circ}}^{90^{\circ}} \frac{d \theta}{\sin \phi}-9\left(\int_{19.5^{\circ}}^{90^{\circ}} \frac{d \theta}{\sin ^{5} \phi}\right)^{2} \\
& <223.960-235.053<0 .
\end{aligned}
$$

vii) If $17.5^{\circ} \leq \gamma \leq 19^{\circ}$, then

$$
g^{\prime}(\gamma) \leq \frac{1}{\sin ^{5} \gamma}\left(h_{1}\left(19^{\circ}\right)+h_{2}\left(17.50^{\circ}\right)\right)<\frac{1}{\sin ^{5} \gamma}(-7.270+7.230)<0
$$

viii) If $15^{\circ} \leq \gamma \leq 17.5^{\circ}$, then

$$
g^{\prime}(\gamma) \leq \frac{1}{\sin ^{5} \gamma}\left(h_{1}\left(17.5^{\circ}\right)+h_{2}\left(15^{\circ}\right)\right)<\frac{1}{\sin ^{5} \gamma}(-7.862+7.365)<0 .
$$

ix) If $0^{\circ} \leq \gamma \leq 15^{\circ}$, then

$$
g^{\prime}(\gamma) \leq \frac{1}{\sin ^{5} \gamma}\left(h_{1}\left(15^{\circ}\right)+h_{2}\left(0^{\circ}\right)\right)<\frac{1}{\sin ^{5} \gamma}(-8.932+7.75)<0
$$

Therefore, $g(\gamma)$ is strictly decreasing on the interval $\left(0^{\circ}, 19^{\circ}\right)$ and is strictly negative on the interval $\left[19^{\circ}, 90^{\circ}\right)$. Also, we can easily see that $g(\gamma)>0$ if $\gamma$ is small enough. We conclude that $g(\gamma)$ has a unique zero at some $\gamma_{0}$. Numerical calculation shows that $\gamma_{0} \approx 14.97^{\circ}$. Furthermore, this numerical calculation of $\gamma_{0}$ is quite accurate since we have shown above that $g(\gamma)$ is strictly decreasing around $14.97^{\circ}$.

Note that $F(\gamma, \gamma)=4 g(\gamma)$. The proof of Theorem 3 is now complete.

\section{A Counter-example.}

We have shown that the family $\{f(\gamma, \gamma)\}$ of stable catenoidal bridges making equal contact angles $\gamma$ with the two plates is connected on the interval of $\gamma$ considered, i.e., if $f\left(\gamma^{\prime}, \gamma^{\prime}\right)$ is in the family of stable catenoidal bridges, so 
is $f\left(\gamma^{\prime \prime}, \gamma^{\prime \prime}\right)$ for all $\gamma^{\prime \prime}$ with $\gamma^{\prime}<\gamma^{\prime \prime}<\pi / 2$. In other words, if a catenoidal

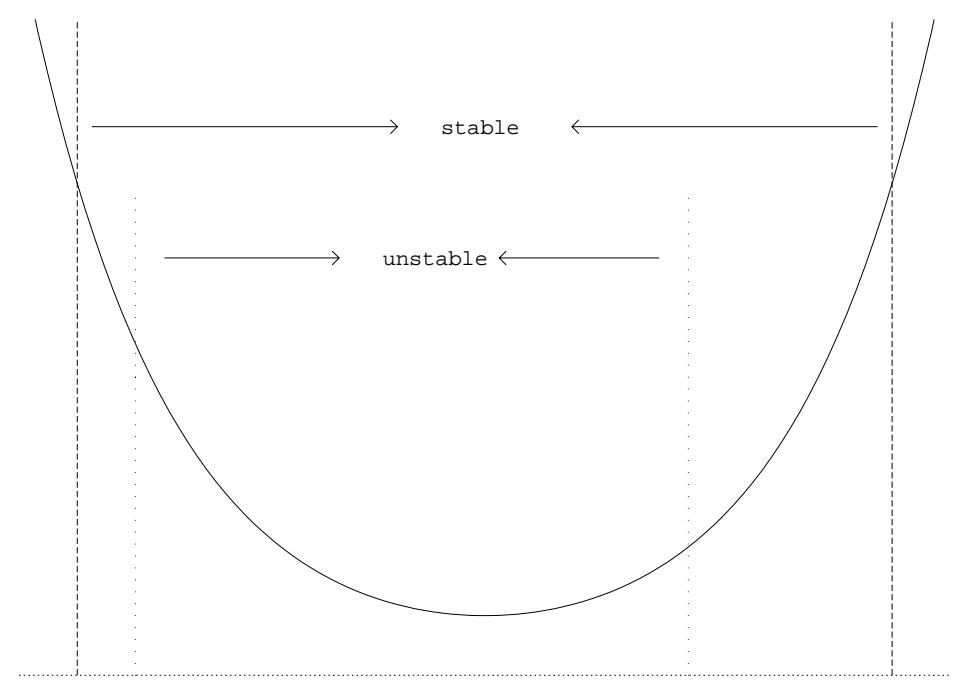

Figure 4. Counter-examples.

bridge making contact angles is unstable, then extending the Delaunay surface (decreasing contact angles but still making equal contact angles) will necessarily result in unstable bridges.

But this is not guaranteed for catenoidal liquid bridges not making equal contact angles. Without loss of generality, we can fix one of the plates and move the other plate. Or equivalently, we can fix $\gamma_{1}$ and let $\gamma_{2}$ vary. We have observed that for the contact angle $\gamma_{1}$ near the critical value $\gamma_{0}$, there exists $\gamma_{2}^{\prime}<\gamma_{2}^{\prime \prime}$ such that $f\left(\gamma_{1}, \gamma_{2}^{\prime}\right)$ is stable but $f\left(\gamma_{1}^{\prime}, \gamma_{2}^{\prime \prime}\right)$ is not stable. In other words, there is an unstable catenoidal liquid bridge with contact angles $\gamma_{1}$, $\gamma_{2}$ and with $\gamma_{1}$ near $\gamma_{0}$ such that extending the Delaunay surface could result in stable liquid bridges. See Figure 4 for instance, it is easily calculated that

1. $F\left(15.0^{\circ}, 15.0^{\circ}\right)<-2.18<0$ which implies that the catenoidal liquid bridge making contact angles of $15.0^{\circ}$ with the plate is stable, and

2. $F\left(16.0^{\circ}, 60^{\circ}\right)>36.29>0$ which means that the catenoidal liquid bridge making contact angles of $16.0^{\circ}$ and $60.0^{\circ}$ with the plates is not stable. See Figure 4.

Finally let us take a closer look at the counter-examples. We have pointed out that the counter-examples are observed when one of the contact angles is near the critical value $\gamma_{0}$. As a matter of fact, it is observed that there are two boundary angles $\gamma^{\prime}$ and $\gamma^{\prime \prime}$, whose numerical values are approximately $14.38^{\circ}$ and $17.62^{\circ}$ respectively, such that 


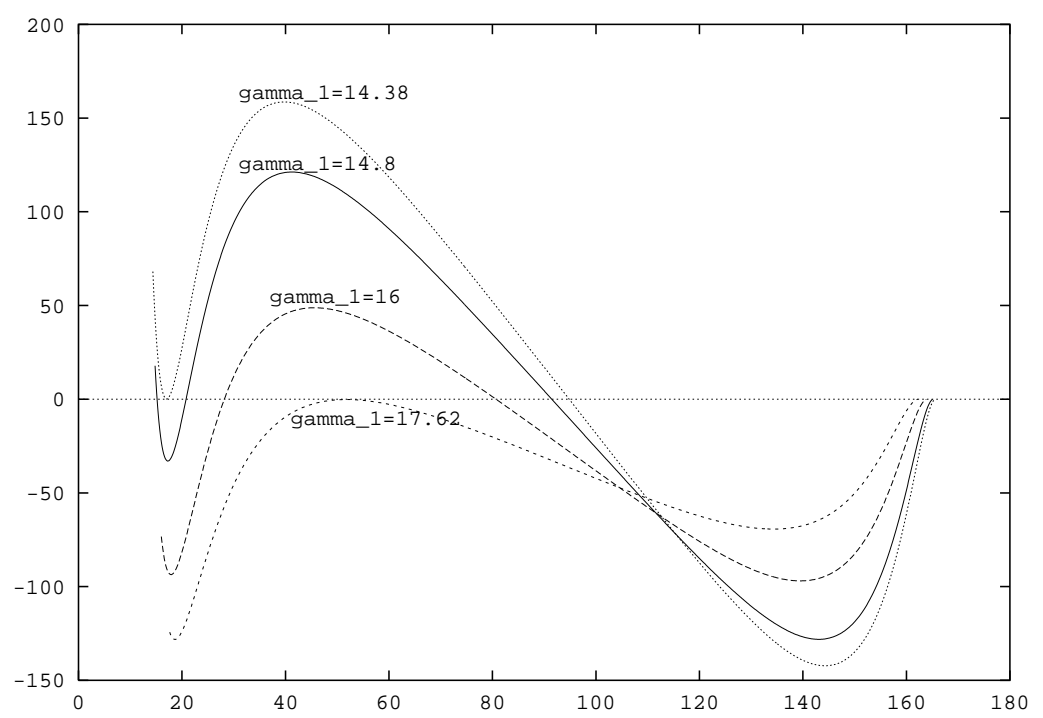

Figure 5. When a Counter-example occurs and when it does not?

- a counter-example occurs if one of the contact angles falls between $\gamma^{\prime}$ and $\gamma^{\prime \prime}$ and

- a counter-example does not occur if neither of the contact angles lies between $\gamma^{\prime}$ and $\gamma^{\prime \prime}$. See Figure 5 .

Furthermore, suppose that we fix the left plate and move the right plate. In other words, we fix $\gamma_{1}$ and let $\gamma_{2}$ vary. With no loss of generality we assume that $0<\gamma_{1} \leq \pi / 2$ and $\gamma_{1}<\gamma_{2}<\pi-\gamma_{1}$, since, otherwise, we could simply switch the role of $\gamma_{1}$ and $\gamma_{2}$. Therefore, we only need to consider the stability of catenoidal liquid bridges when the right plate is very close to the left plate at first and moves away from the left plate until it reaches a position where it makes the same contact angle as the left plate. The stability and instability of catenoidal liquid bridges can be completely described as follows:

1. if $\gamma_{1}>\gamma^{\prime \prime}$, then the catenoidal bridges remain stable from the beginning (when $\gamma_{2}=\pi-\gamma_{1}$ ) to the end (when $\gamma_{2}=\gamma_{1}$ );

stable

2. if $\gamma_{0}<\gamma_{1}<\gamma^{\prime \prime}$, then the catenoidal bridges are stable in the beginning when the right plate is close to the left plate and remains stable until it reaches a certain position. It then becomes unstable for a while and it will be stable again until the very end; 


stable unstable stable

3. if $\gamma^{\prime}<\gamma_{1}<\gamma_{0}$, then the catenoidal bridges are stable in the beginning when the right plate is close to the left plate and remains stable until it reaches a certain position. It then becomes unstable for a while and it will be stable again, but only for a while. It will turn unstable again until the very end;

stable unstable stable unstable

4. if $\gamma_{1}<\gamma^{\prime}$, then the catenoidal bridges are stable in the beginning when the right plate is close to the left plate and remains stable until it reaches a certain position. It becomes unstable until the very end.

stable unstable

\section{References}

[1] M.Athanassenas, A variational problem for constant mean curvature surfaces with free boundary, J. Math., 377 (1987), 97-107.

[2] W.C.Carter, The forces and behavior of fluids constrained by solids, Acta Metall., 36 (8) (1988), 2283-2292.

[3] R. Courant and D. Hilbert, Methods of Mathematical Physics, 1, John Wiley \& Sons, New York, 1989, 397-424.

[4] D.E. Delaunay Sur la surface de révolution dont la courbure est constante, J. Math. Pures Appl., 6 (1841), 309-315.

[5] R. Finn Equilibrium Capillary Surfaces, Springer-Verlag, New York, 1986.

[6] Nonuniqueness and uniqueness of capillary surfaces, Manuscr. Math., 61 (1988), 347-372.

[7] and T.I. Vogel On the volume infimum for liquid bridges, Zeit. Anal. Anw., 11 (1) (1992), 3-23.

[8] D. Langbein Stability of liquid bridges between parallel plates, preprint, 1-20.

[9] J.W.S. Rayleigh On the capillary phenomena of jets, Scientific Papers, 1, Cambridge University Press, 1899, 433-442.

[10] D. Strube Stability of a spherical and a catenoidal liquid bridge between two parallel plates in the absence of gravity, Microgravity Sci. Technol., 4 (1991), 104.

[11] Stability of a spherical and a catenoidal liquid bridge between two parellel plates in the absence of gravity - Correction of a Paper [10] by D. Strube, Microgravity Sci. Technol., 1, (1992), 56-57.

[12] T.I. Vogel Stability of a liquid drop trapped between two parallel planes, SIAM J. Appl. Math., 47 (1987), 516-525.

[13] Stability of a liquid drop trapped between two parallel planes II: General contact angles, SIAM J. Appl. Math., 49 (1989), 1009-1028. 

technical report (March 1991), 1-5.

[15] L. Zhou On the volume infimum for liquid bridges, Zeit. Anal. Anw., to appear

Received May 25, 1993 and revised October 18, 1993. This work is supported by NASA under Grant NAG3-1143.

JOHNS HOPKINS UNIVERSITY

BALTimoRE, MD 21286

E-mail address: lianmin@math.jhu.edu

\section{Editor's Note:}

Following a quietus of half a century, the last decade or so has witnessed a surge of interest on liquid free-interface problems, and notably on determination of stability criteria. In the engineering as well as in mathematical literature, numerous authors have investigated the "static stability" of particular configurations. In all such publications known to me (including one that bears my name), the criterion for stability has been taken to be positivity of the quadratic form arising from the second variation of the energy functional on the extremal surface considered. In this respect the foregoing paper is no exception, and its usage is certainly consistent with that of a significant body of literature by respected authors.

From a formal mathematical point of view, the matter reduces to definition of the term "static stability". But it should be emphasized that positivity of the second variation on an extremal does not in itself guarantee even a weak local minimum for the functional considered; thus in a physical configuration for which the functional is mechanical energy, the published literature has not yet provided proofs that the energy is locally minimized, even among surfaces that are pointwize close to the extremal together with their derivatives.

In the context of the classical one-dimensional fixed endpoint problem, we may consider as an example the functional

$$
\begin{aligned}
\mathcal{I}[y] & \equiv \int_{0}^{1} f\left(x, y, y^{\prime}\right) d x, \\
f & =x^{4} e^{-\frac{16}{x}} y^{\prime 2}-y^{3}
\end{aligned}
$$

with $\mathcal{I}$ to be minimized (locally) among piecewise smooth functions $y(x)$ for which $y(0)=y(1)=0$. One finds immediately that the function $y(x) \equiv 0$ provides an extremal on which $\mathcal{I}=0$, and if one considers any family of functions $y(x ; \varepsilon)$ such that $y(x ; 0) \equiv 0, y(0 ; \varepsilon)=y(1 ; \varepsilon)=0$, then the second 
variation on the given extremal becomes

$$
\ddot{\mathcal{I}}(0)=2 \int_{0}^{1} x^{4} e^{-\frac{16}{x}} \eta^{\prime 2} d x, \quad \eta=\dot{y}(x ; 0)
$$

which is positive for any $\eta$ that achieves the boundary data and doesn't vanish identically.

Nevertheless, the function $y \equiv 0$ does not provide a local minimum for $\mathcal{I}$. The family of functions

$$
y(x ; \varepsilon)=\left\{\begin{array}{cc}
0, & x=0 \\
e^{-\frac{1}{x}} e^{-\frac{1}{\varepsilon-x}}, & 0<x<\varepsilon \\
0, & \varepsilon \leq x \leq 1
\end{array}\right\}
$$

converges uniformly to zero in the closed interval together with its derivatives of every order as $\varepsilon \rightarrow 0$, but is easily seen to yield a negative value of $\mathcal{I}$ for all positive but sufficiently small $\varepsilon$.

Still simpler examples can be given, which yield convergence to the extremal together with derivatives up to any prescribed order.

In the situation studied in the foregoing paper, and presumably also for most problems considered in earlier literature, it should be expected in view of the particular nature of the problem and of the considered extremals that the stability criterion in terms of second variation actually provides a local energy minimum, even in the strong sense of the Calculus of Variations (which does not require derivatives to be close to those of the extremal). In fact, some years ago in an unpublished report of the Max-Planck-Institut in Bonn, H.C. Wente did provide a framework for a proof of the strong local minimizing property in connection with his stability criteria for pendent liquid drops; later, U. Patnaik in an yet unpublished dissertation at University of Toledo provided a proof of that property in another particular context. These seem to have been the only authors to have taken cognizance of the question. Presumably, a reasoning along lines as indicated by these authors would apply to the configurations examined in the paper just preceding. In my view, such a step would be essential for justifying the results conceptually as theorems on stability. In the interim, the contribution presently offered by the foregoing paper seems to me striking and of interest in itself; the paper received a strongly favorable referee report with which I am in accord, and I am pleased that it could be published by the Pacific Journal.

Robert Finn 